\title{
Cascading nonlinearities in an organic single crystal core fiber: the Čerenkov regime
}

\author{
William E. Torruellas, Gijs Krijnen, Dug Y. Kim, Roland Schiek, George I. Stegeman \\ C.R.E.O.L. University of Central Florida, Orlando, FL 32826. USA
}

Petar Vidakovic, Joseph Zyss

Centre National d'Etudes des Telecommunications, 196 Av. Henri Ravera, 92220 Bagneux, France

Received 14 April 1994; revised manuscript received 6 July 1994

\begin{abstract}
The large nonlinear phase shifts imparted to the fundamental beam during Cerenkov second harmonic generation (SHG) in a DAN, 4-(N,N-dimethylamino)-3-acetamidonitrobenzene, single crystal core fiber are explained and modelled numerically. Cascading upconversion and downconversion processes leads to nonlinear phase shifts produced by the second order nonlinear coupling of the guided fundamental mode and the component of the Cerenkov second harmonic field trapped in the fiber cladding.
\end{abstract}

\section{Introduction}

The possibility of a cascaded second order effect interfering with the intrinsic third order polarization has been known and understood since the early days of nonlinear optics [1]. For instance Flytzanis proposed the enhancement of third harmonic generation in crystal structures without inversion symmetry, followed by Meredith's experimental demonstration [2]. In three-wave-mixing enhanced polariton-wave creation near phase matching was also demonstrated, allowing for the magnitude and sign of the intrinsic third order susceptibility to be measured in GaAs [3]. Recently a vigorous renewed interest in this subject has been generated by advances in the magnitude of the second order nonlinearity which can be used for relatively efficient second harmonic generation. The cascaded process can now produce effects orders of magnitude larger than those due to the native third order susceptibility of non-centrosymmetric mate- rials [4-11]. In addition the possibility of solitonlike propagation, all-optical switching devices, and perhaps even all-optical transistor action has been numerically explored, opening very exciting areas of research [12-15].

Cascaded second order effects can occur in organic materials in a number of ways. For example, the possibility of such effects enhancing the second order molecular hyperpolarizability via the retardation of local fields has been proposed in isotropic, i.e. centrosymmetric media [16-19]. However, using propagation effects in non-centrosymmetric single crystals or poled polymers with very large phase matchable second order susceptibilities appears as a more promising route [20-22]. These latter material systems are therefore natural candidates for the observation of very large effects via the cascading of two second order processes. The strong field confinements (i.e. high intensities at low input powers) provided by single crystal core fibers recently fabricated 
for SHG into the blue-green spectral region provide an interesting option for low power all optical applications [23]. In fact very large spectral broadenings due to self-phase modulation have already been reported in DAN single core fibers and have been used to demonstrate pulse compression [24]. The physical origin of these large effective nonlinearities had been unknown until we recently identified it to be induced by the cascading of second harmonic generation and down conversion [25]. In our previous paper we clearly showed the cascading mechanism to be the dominant one by four distinct nonlinear optical techniques, third harmonic generation, self-phase modulation, nonlinear interferometry and Z-scan. Although we did observe the necessary dependence of the phase shifts on the presence of second harmonic light in the fiber, nonlinear phase shifts were observed only when SHG was detected, we also found that the second harmonic of a $1.320 \mu \mathrm{m} \mathrm{Nd:YAG}$ laser could not be guided by the DAN fiber core because the index dispersion results in a refractive index of the core smaller than that of the cladding. This raised interesting questions about the waveguide cascaded process which had only been observed previously in geometries in which the fundamental and second harmonic were guided modes of a particular sample or geometry. In this communication we demonstrate that the observed large nonlinear phase shifts are produced by the presence of an experimentally observed strong second harmonic wave, the part of the Cerenkov field which exists in the fiber core.

\section{Summary of experimental results}

The crystal geometry of the DAN single crystal in the core of the fiber is shown in Fig. 1. A summary of the experimental conditions is also presented in $\mathrm{Ta}$ ble 1. There are two possible orthogonal normal modes for propagation along the fiber axis, one polarized in the $Y$-plane and the second in the $X-Z$ plane. Whether guiding is allowed or not for a given polarization is determined by the refractive index of the SF1 glass cladding, relative to that of the core for that polarization. Fortunately there is sufficient information available to estimate the index dispersion in both the cladding and core material. These results are shown in Fig. 1. Note that in our case the DAN

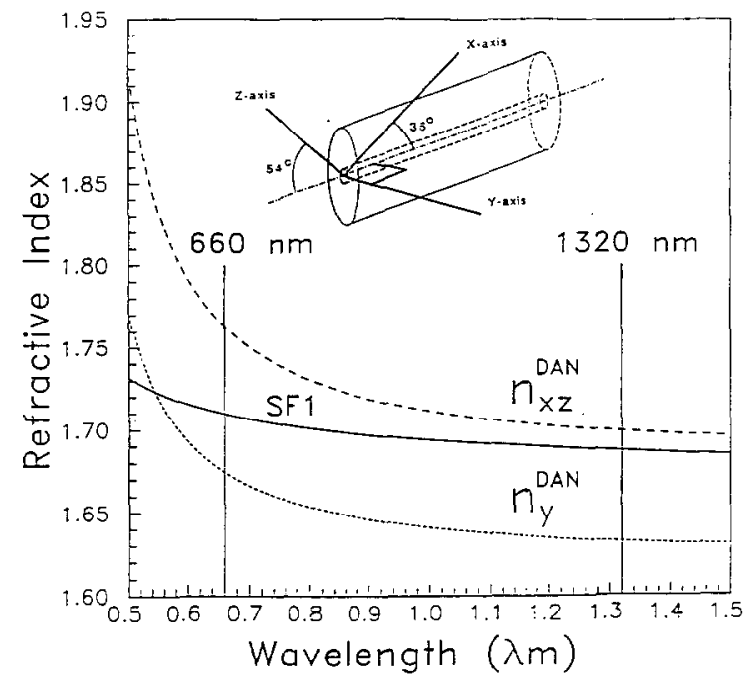

Fig. 1. DAN fiber with the crystal orientation in the fiber core [5]. Refractive index dispersion for polarization along $X^{\prime}$, in the $X Z$ plane, and $Y$ in the DAN crystal core and for glass SF1 [26]. The refractive indices at $1320 \mathrm{~nm}$ and its second harmonic are emphasized, showing the impossibility of the existence of a true guided mode at second harmonic along the $Y$ axis for a SF1/DAN fiber.

fiber does not support waveguiding polarized along the $Y$ axis at either the fundamental $(1320 \mathrm{~nm})$ or second harmonic ( $660 \mathrm{~nm}$ ) wavelengths used in our experiment. It was designed for efficient Čerenkov type SHG emission for a fundamental guided mode cxcited in the $X-Z$ planc and second harmonic polarized along the $Y$-axis. Indeed we found that we could not propagate a $Y$-polarized mode at $1320 \mathrm{~nm}$ in the fiber. An $X-Z$ polarized mode did propagate. When a randomly polarized $\mathrm{HeNe}$ laser beam $(632 \mathrm{~nm})$ was launched into the fiber, the only guided beam at the output of the fiber was also polarized along the $X-Z$ plane. The possibility of the refractive index of the fiber core along the $Y$ axis, shown in Fig. 1, being larger than that of the SF1 cladding can then be excluded [26]. In addition the only large second order tensor element in the fiber geometry of Fig. 1 corresponds to type I phase matching, with the fundamental wave guided in the $X-Z$ plane with a fundamental field polarization perpendicular to the second harmonic polarized along $Y$. Therefore the only possible second harmonic light generated has to be radiated out of the core of the fiber, i.e. Cerenkov SHG.

The spatial distribution and polarization properties of the observed second harmonic beams were 
Table 1

Summary of experimental conditions

\begin{tabular}{llll}
\hline Cladding material & Core material & $E_{y}^{(2 \omega)}$ & $E_{x^{\prime}}^{(\omega)}$ \\
\hline SF1 & DAN & Cerenkov type & $30 \mathrm{~W}$ \\
I mm O.D & $5 \mu \mathrm{m}$ core diameter & Polarized along $Y$ & $\begin{array}{l}\text { Polarized along } X^{\prime} \\
(X-Z \text { plane })\end{array}$ \\
\hline
\end{tabular}

measured at the output of the fiber. The second harmonic light was clearly polarized at $90^{\circ}$ from the fundamental. Images of the radiation emerging from the end of the fiber are shown in Fig. 2. When the fundamental mode was excited at $1320 \mathrm{~nm}$, the most intense second harmonic light was observed to be $Y$ polarized and to emerge from the fiber core with a fundamental-like spatial profile. Similarly, when the fundamental was launched into the first higher order spatial mode, again the most intense second harmonic light was observed to emanate from the fiber core, with a spatial profile similar to that of the fundamental and polarized along the $Y$ axis. In addition, the power of the second harmonic light emerging from the fiber core grew quadratically with input power. Therefore, contrary to expectations, Fig. 1, a $Y$-polarized second harmonic mode seemed to be guided along the fiber. We also observed that the SHG beam pattern did not change with fundamental power, excluding any spatial-soliton-like effects leading to guiding in the core.

The total amount of SHG collected at the end of
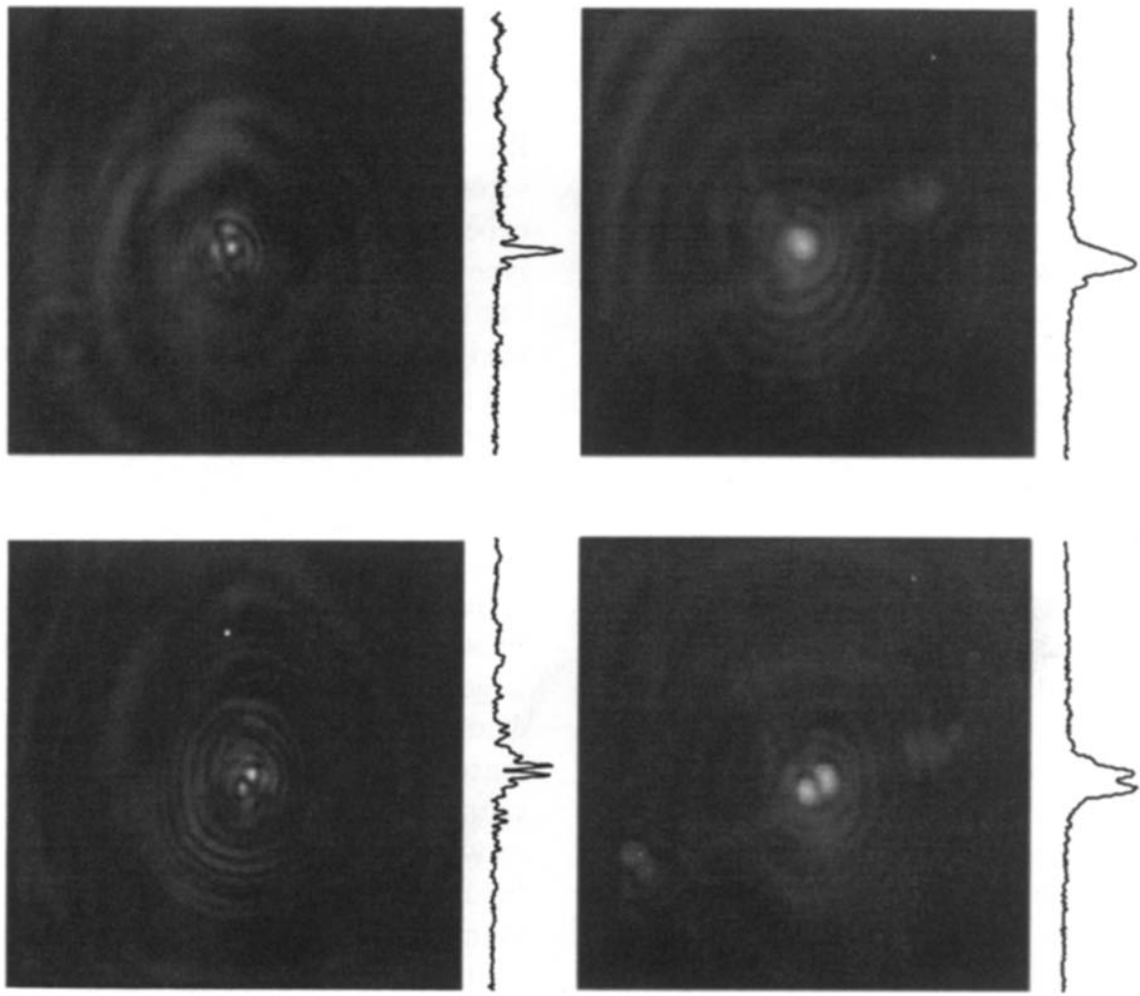

Fig. 2. Mode patterns of the two lowest order modes for both the fundamental and the second harmonic at the exit face of the DAN fiber. The two figures on the right show the SHG fields profiles collected at the end of the fiber. The figures on the left show the profiles of the two lowest order modes at the fundamental wavelength of $1320 \mathrm{~nm}$. 
the fiber was also measured. Even though the most intense SHG came from the core of the fiber, it only represented about $3 \%$ of the total SHG gathered from the fiber, i.e. the integrated second harmonic power radiated into the cladding was 31 times larger than the core portions shown in Fig. 2. The latter observation is not surprising because under Čerenkov type radiation most of the SHG is radiatcd transverscly into the cladding whose cross-sectional area is orders of magnitude larger than that of the core. However at a given propagation position the SHG intensity is strongest in the core.

To date, coupled mode equations for which the fundamental and second harmonic copropagate have been used in analyzing the cascading effect. Clearly, they do not apply in a simple way to the current geometry. In the rest of this paper we discuss the results of numerical modelling of our geometry and the insights gained into cascading based on Čerenkov SHG. We will make an attempt to explain: (i) the field profiles observed at the end of the fiber, and (ii) the magnitude and sign of the measured nonlinear phase shifts.

\section{Cerenkov SHG in a symmetric planar waveguide, a qualitative picture}

From the experimental observations discussed above, it is clear that the nonlinear mixing between the second harmonic field bound to the core and the guided wave fundamental field must be responsible for the measured nonlinear phase shift. This is reminiscent of the calculation of Flytzanis in which he first separated the solution for the second harmonic into its inhomogeneous and homogeneous parts, and then examined the effect of the inhomogeneous part on the fundamental propagation wavevector [11].

$$
\begin{gathered}
\nabla x \nabla x E_{y}^{2 \omega}-\frac{\epsilon_{y}(2 \omega)(2 \omega)^{2}}{c^{2}} E_{y}^{2 \omega} \\
=\frac{(2 \omega)^{2}}{c^{2}} \frac{\chi_{y x^{\prime} x^{\prime}}^{(2)}(-2 \omega ; \omega, \omega)}{2} E_{x^{\prime}}^{\omega} E_{x^{\prime}}^{\omega}, \\
\nabla x \nabla x E_{x^{\prime}}^{\omega}-\frac{\omega^{2} \epsilon_{x^{\prime}}(\omega)}{c^{2}} E_{x^{\prime}}^{\omega} \\
=\frac{\omega^{2}}{c^{2}} \chi_{x^{\prime} x^{\prime} y^{\prime}}^{(2)}(-\omega ; 2 \omega,-\omega)\left(E_{x^{\prime}}^{\omega}\right)^{*} E_{y}^{2 \omega} .
\end{gathered}
$$

Based on the preceding nonlinearly coupled propagation equations, where $X^{\prime}$ refers to the polarization direction in the " $X-Z$ ' plane where type I phase matching can occur, Fig. 1, and following Flytzanis's approach the change in the propagation constant due to cascading is

$\delta k_{x^{\prime}}^{\omega}=-\frac{8 \pi^{2} d_{\mathrm{eff}}^{2}}{c \epsilon_{0} n_{y}^{2 \omega} n_{x^{\prime}}^{\omega}} \frac{z}{\lambda^{2}\left[\left(k_{y}^{2 \omega}-2 k_{x^{\prime}}^{\omega}\right) z\right]} I^{\omega}$

Here, $d_{\mathrm{eff}}$ is the effective nonlinear coefficient, for example $27 \mathrm{pm} / \mathrm{V}$ in the case of DAN, $I^{\omega}$ is the fundamental intensity and $\lambda$ is the wavelength of the fundamental beam. This wavevector shift leads to a nonlinear phase shift with propagation distance, qualitatively explaining the experimental observation (ii).

Our situation is more complex because the overlap of the harmonic fields with the fundamental is actually very small, being limited to the fiber core. Although guided normal modes do not exist for the second harmonic field, strong SHG is achieved via the so-called Čerenkov phase-matching technique. In such geometries the following condition has to be satisfied $n_{\mathrm{cl}}(2 \omega)>N_{\mathrm{efr}}(\omega)$ so that the Cerenkov radiation angle into the cladding is given by $\theta_{\text {cer }}=$ $\cos ^{-1}\left[N_{\text {eff }}(\omega) / n_{\mathrm{cl}}(2 \omega)\right]$, where $n_{\mathrm{cl}}(2 \omega)$ and $N_{\mathrm{eff}}(\omega)$ are respectively the cladding refractive index at the harmonic frequency and effective refractive index of the guided mode at the fundamental wavelength. The detailed analysis in fiber geometries is very complicated because of the mathematics associated with cylindrical functions and satisfying boundary conditions [27].

There are two interesting limits for Čerenkov radiation. For the harmonic field in the core, the propagation effective index is automatically given by $N_{\text {eff }}(\omega)$. If $n_{\text {co }}(2 \omega)>N_{\text {eff }}(\omega)$ where $n_{\text {co }}(2 \omega)$ is the core refractive index at the harmonic frequency, the propagation wavevector normal to the fiber axis in the core is real. The other regime occurs for $N(\omega)>n_{\mathrm{co}}(2 \omega)$ so that the wavevector normal to the surface is imaginary and the fields decay exponentially from the center of the core to the boundaries. This component of the Cerenkov field in the core grows with propagation distance until a steady state balance between radiation loss and growth is reached 
(except for the slow depletion of the fundamental with distance).

Steady state analytical solutions are available for the fields in symmetric slab waveguides for $n_{\mathrm{co}}(2 \omega)>N_{\text {eff }}(\omega)$ [28]

$$
\begin{aligned}
E_{y}^{2 \omega}= & F \exp \left(\mathrm{i} \rho_{2 \omega} x^{\prime}\right), \text { for } x^{\prime} \geq a / 2 \\
= & F^{\prime} \cos \left(\kappa_{2 \omega} x^{\prime}\right) \\
& -2 A^{2}(\omega / c)^{2} d_{y x^{\prime} x^{\prime}}\left(\frac{\cos \left(2 \kappa_{\omega} x^{\prime}\right)}{\kappa_{2 \omega}^{2}-4 \kappa_{\omega}^{2}}+\frac{1}{\kappa_{2 \omega}^{2}}\right),
\end{aligned}
$$

$$
\text { for } x^{\prime} \leq a / 2 \text {. }
$$

Here $A$ is the amplitude of the fundamental mode in the core; $\kappa$ and $\rho$ are the transverse components of the propagation constants in the core and cladding, respectively. The field boundary conditions at the interface core-cladding have to be fulfilled leading to both $F$ and $F^{\prime} \propto A^{2}$. One can interpret this type of Čerenkov SHG as being a leaky wave which is continuously regenerated by the second harmonic polarization. However we should emphasize that such an ansatz is only valid at the so called Cerenkov phasematching, it cannot match the boundary conditions at the entrance face and is only applicable to the low depletion regime where back coupling with the fundamental is omitted. In order to solve such a problem a generalized approach has been taken in the past [ 30,31$]$. In addition the solution presented here is only applicable for geometries where the core refractive index at the second harmonic is larger than the effective refractive index of the guided mode at the fundamental wavelength, $N_{\text {eff }}(\omega)<n_{\text {co }}(2 \omega)<$ $n_{\mathrm{cl}}(2 \omega)$.

According to Sellmeier's equations [26], this is not the case of our fiber for which $n_{\mathrm{cl}}(2 \omega)>$ $N_{\text {eff }}(\omega)>n_{\text {co }}(2 \omega)$. The major change in the steady state solutions from the preceding case will be that the harmonic field in the core will be given by hyperbolic functions. In both limits these solutions seem to have all the ingredients to explain the first part of the phenomena we are exploring (i): a strong SHG field trapped in the core of the waveguide with good overlap with the mode propagating at the fundamental frequency explaining the observed radiation of Fig. 2. However, because these solutions do not include the effect of the harmonic field back on the fundamental, we do not proceed further with them. This additional complication which is crucial to producing nonlinear phase shifts on the fundamental encouraged us to approach this problem numerically.

\section{Numerical solutions}

In order to gain a more complete picture of this second order process, we performed a numerical simulation based on an enhanced finite difference beam propagation method, EFDBPM, with improved accuracy of the propagation constants [32]. This method takes into account diffraction of both the fundamental and the nonlinearly generated second harmonic fields. It alternates linear EFDBPM calculations at the fundamental and second harmonic followed by a step in which the coupling between electric fields at both wavelengths is taken into account, and is referred to as SHGBPM [33]. Under the slowly varying envelope approximation (SVEA) and discretising the fields in the transverse direction, $X^{\prime}$ for the fundamental and $Y$ for the second harmonic, Eqs. (1a) and (1b) can be rewritten as follows

$$
\begin{aligned}
& \mathrm{i} \frac{\partial \boldsymbol{E}_{y}^{2 \omega}}{\partial z}=\mathbf{M}^{2 \omega} \boldsymbol{E}_{y}^{2 \omega}+\frac{1}{4 n_{0} k_{0}} \frac{\partial^{2} \boldsymbol{E}_{y}^{2 \omega}}{\partial z^{2}}+\frac{k_{0} \chi_{y x^{\prime} x^{\prime}}^{(2)}}{n_{0}}\left(\boldsymbol{E}_{x^{\prime}}^{\omega}\right)^{2}, \\
& \mathrm{i} \frac{\partial \boldsymbol{E}_{x^{\prime}}^{\omega}}{\partial z}=\mathbf{M}^{\omega} \boldsymbol{E}_{x^{\prime}}^{\omega}+\frac{1}{2 n_{0} k_{0}} \frac{\partial^{2} \boldsymbol{E}_{x^{\prime}}^{\omega}}{\partial z^{2}}+\frac{k_{0} \chi_{y x^{\prime} x^{\prime}}^{(2)}}{n_{0}}\left(\boldsymbol{E}_{x^{\prime}}^{\omega}\right) \boldsymbol{E}_{y}^{2 \omega} .
\end{aligned}
$$

Here $k_{0}=2 \pi / \lambda_{0}$ and $n_{0}$ is a suitable background refractive index. $E_{y}^{2 \omega}$ and $E_{x^{\prime}}^{\omega}$ are vectors representing the discretised field envelopes and $\mathbf{M}^{\omega}$ and $\mathbf{M}^{2 \omega}$ are tridiagonal discretisation matrices. The equations are solved using a split-step approach; the first two linear operators are handled with the EFDBPM for each wavelength whereas the nonlinear operator is solved pointwise for all discretisation points. It is a two-dimensional scalar calculation on a slab waveguide geometry which contains the relevant physics in a simple geometry. We do not need to take into account vector diffraction because our polarizations are wcll defined and no additional birefringence along the fiber is observed. Going to cylindrical coordinates, the DAN fiber case, may affect slightly our results in an absolute sense but not in a qualitative way, which is our only goal at this point.

The first case examined numerically corresponded to the experimental situation refractive indices etc., 
as estimated from the reported Sellmeier coefficients [26]. Fig. 3a shows the evolution of the second harmonic field along the slab. The refractive index profile chosen is shown in Fig. $3 \mathrm{~b}$ as well as the profiles of the fundamental and second harmonic fields. We used a propagation distance of $4.28 \mathrm{~mm}$ and an input fundamental peak power of $30 \mathrm{~W}$ which match our experimental conditions. Even though the mode profile matches our observation (i), shown in Fig. 2, in this case the nonlinear phase shift was calculated to be small, $\pi / 10$ radians, and positive as compared to $-\pi / 4$ in our experiment.

A series of calculations using the same numerical approach was then performed to try to reproduce the experimental observations (i) and (ii). Since the structure of the core fields changes at $n_{\mathrm{co}}(2 \omega)=N_{\text {eff }}(\omega)$, we varied the parameter $n_{\mathrm{co}}(2 \omega)-N_{\mathrm{eff}}(\omega)$. The results of such calculations are presented in Fig. 4. The situation just discussed, case 1 , shows a small depletion $(<5 \%)$ and a positive nonlinear phase shift. As the refractive index of the core was increased, larger phase shifts could be achieved at the expense of an increased depletion of the fundamental. It is worth noting at this point that almost complete depletion and a zero phase shift are both obtained for a small positive refractive index difference, Fig. 4. A large negative phase shift is obtained for yet larger positive values. Such a numeri-
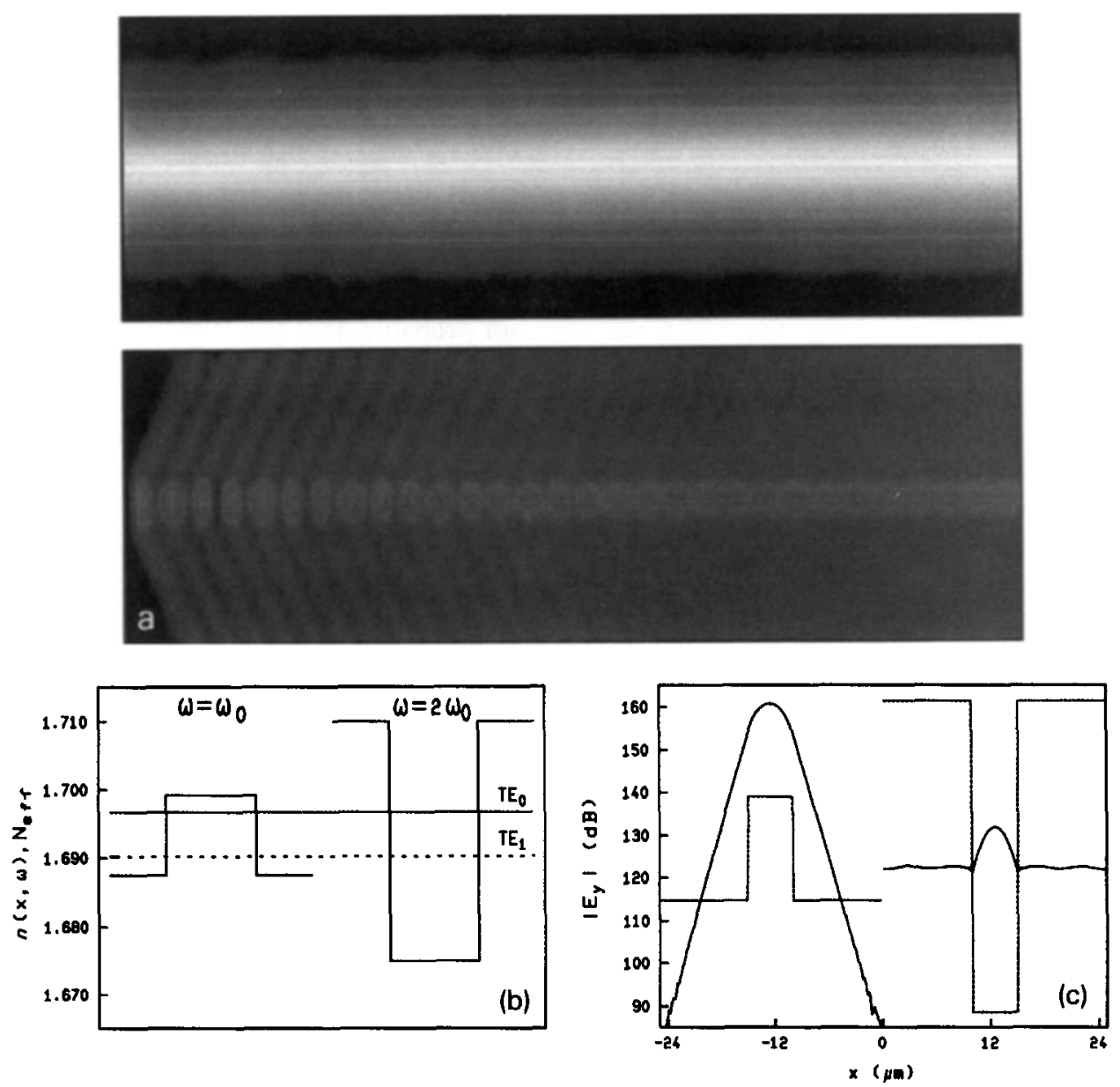

Fig. 3. (a) SHGBPM results for the $\mathrm{TE}_{0}$ mode of a slab waveguide with similar dimensions and refractive indices as the DAN fiber, predicted from the Sellmeir's equations of Ref. [26]. The intensities of the fundamental (top) and SHG (bottom) are shown with a 60 $\mathrm{dB}$ gray scale. (b) The assumed refractive index profile of the fiber and the effective refractive indices of the allowed modes at the fundamental frequency are also shown. (c) Amplitudes of the fundamental and SHG beams at the output end of the slab waveguide. 

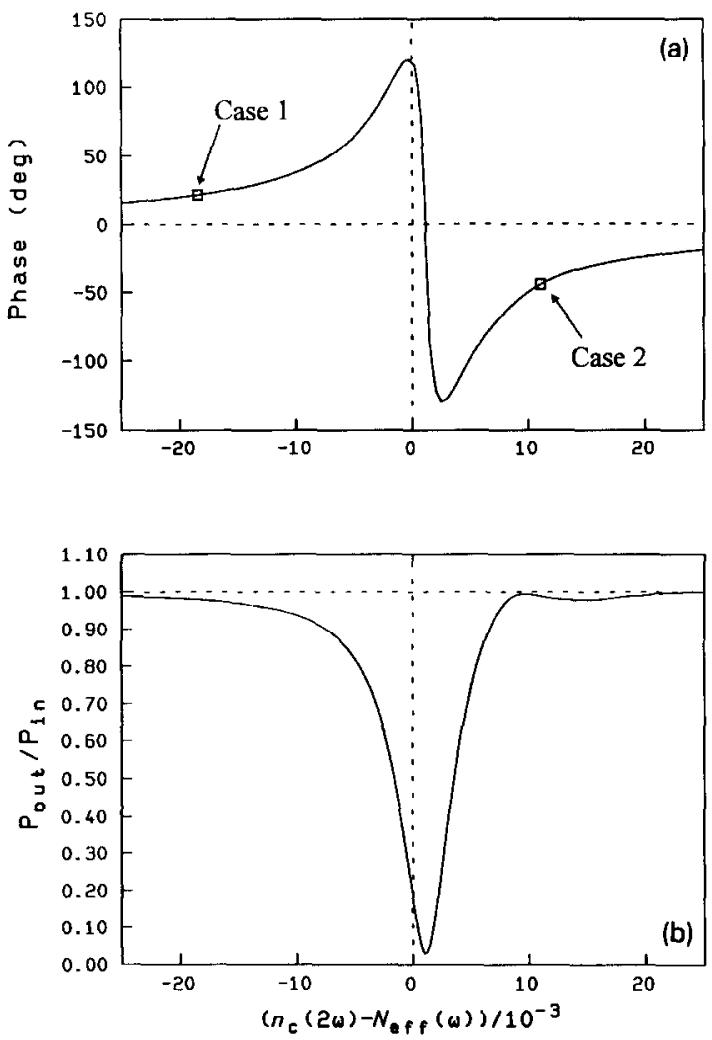

Fig. 4. (a) Nonlinear phase shift induced on the $T E_{0}$ mode by the cascading process versus refractive index detuning, for $30 \mathrm{~W}$ of fundamental input power. (b) Depletion of the fundamental wave versus refractive index detuning. The above curves are predictions of our SHGBPM calculation. Case 1 refers to Fig. 3 and case 2 to Fig. 5 .

cal approach shows that large conversions to the harmonic can be achieved, as well as very large nonlinear phase shifts using Čerenkov radiation, by carefully tailoring the effective refractive indices involved. The SHGBPM [33], also proves itself here as a formidable design tool for future waveguide optimizations.

It seems that $n_{\mathrm{co}}(2 \omega)>n(\omega)$ is needed for a negative phase shift, the result that we observed experimentally. The results of a calculation which closely mimics our fiber experimental results, case 2 , are shown in Fig. 5. The film thickness is chosen equal to the fiber diameter and the refractive index profile at the fundamental frequency is the same as for the fiber case. The core refractive index at $2 \omega$ was adjusted until the calculated and observed nonlinear phase shifts were in agreement both in magnitude and sign, $-\pi / 4$. The calculated fraction of fundamental power converted into the harmonic power was in that case a few percent, $(\sim 5 \%)$, also in good agreement with experiment. Similar results can be obtained by adjusting the core diameter instead of the core index at the harmonic wavelength. Varying either of those two parameters tunes the mismatch of the propagation constants, allowing for positive and negative nonlinear phase shifts. In this simulation the induced nonlinear phase distortion for the fundamental is $-\pi / 4$, fitting our experimental results as measured by self-phase-modulation and nonlinear interferometry for 30 watts of fundamental power in the DAN fiber. Although most of the second harmonic power is in the radiation field in the cladding, it is evident from Fig. 5 that the most intense SH field occurs in the fiber core. The Cerenkov radiation field is also obvious.

The most puzzling element of our calculation is the large refractive index change necessary to match our experimental results, case 2 of Fig. 4 . The best fit was obtained for a modified core refractive index at the second harmonic of 0.031 from that predicted by the Sellmeier's equations, Fig. 1 [26]. It is possible, but not probable, that this difference reflects the weak. nesses of our model: a CW calculation with a single transverse dimension considered in a planar geometry where the core width was assumed to be equal to that of the existing DAN/SF1 fiber. It is also possible that the refractive indices assumed are not appropriate for our particular fiber. We note at this point that such an increase in refractive index was previously observed in similar fibers and attributed to an increase of the packing density of the DAN single crystal when grown in glass capillaries [34].

\section{Summary}

In conclusion, we have shown by a numerical simulation that the DAN fiber designed for efficient $\nearrow_{\text {er- }}$ enkov radiation is capable of producing large nonlinear phase shifts by cascading of up- and downconversion processes. Even though the refractive index of the fiber core is less than that of the cladding, intense SHG waves copropagating with the modes at the fundamental frequency interact with the latter via down-conversion, shifting the phase of the field at the fundamental frequency by $-\pi / 4$ in just a few milli- 

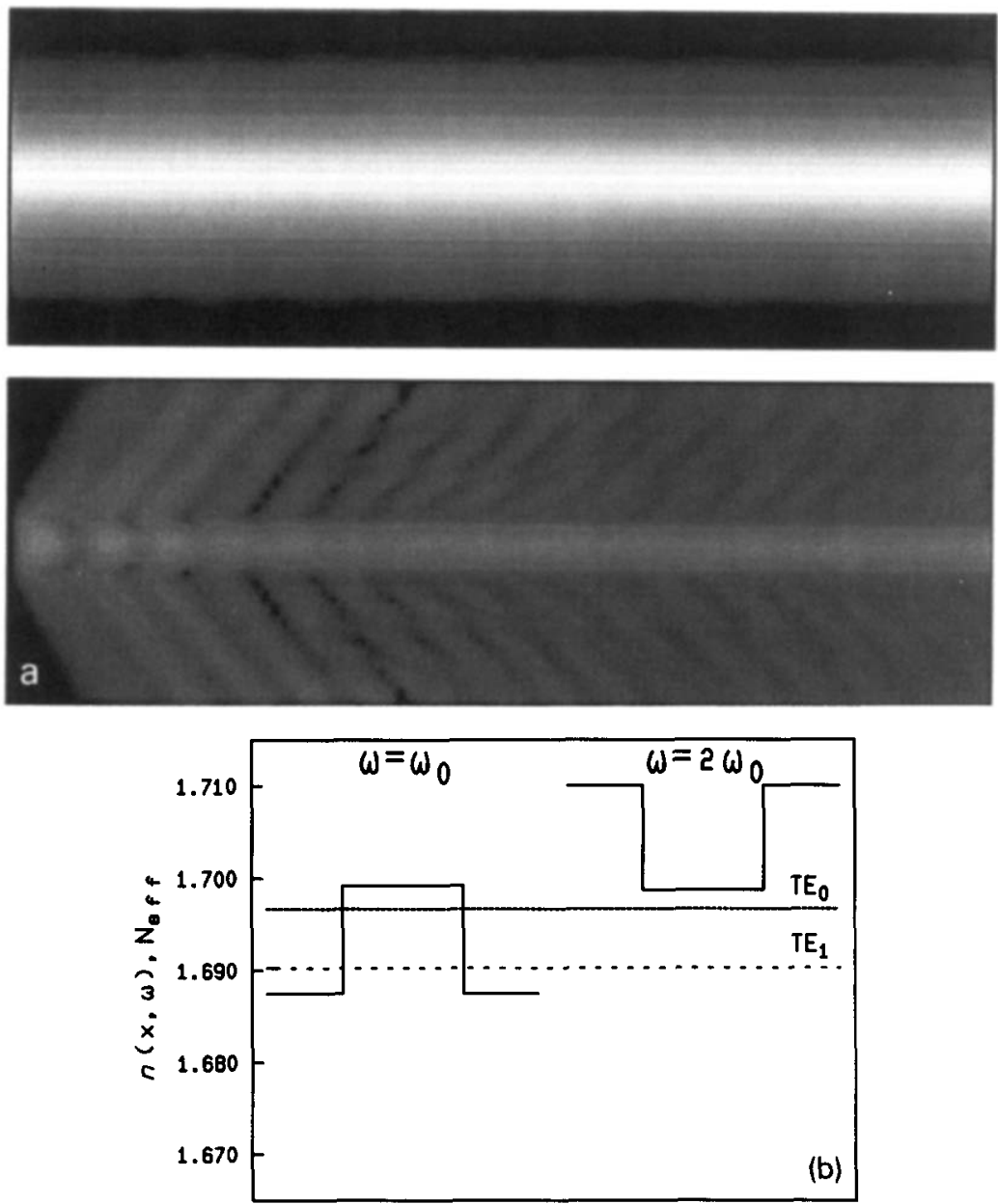

Fig. 5. (a) The results of the SHGBPM are shown for both the mode at the fundamental wavelength (top), and the generated Cerenkov second harmonic field (bottom), the intensities are represented with a $60 \mathrm{~dB}$ gray scale. (b) Slab refractive index profile used to model Cerenkov SHG in the DAN fiber. The refractive index of the core at the second harmonic frequency was increased by 0.031 relative from the predictions of the Sellmeir's equations to reproduce the ratio of radiated to guided second harmonic power and the magnitude and sign of the nonlinear phase shift of the mode at the fundamental wavelength [26].

meters of fiber with peak input intensities below 50 $\mathrm{MW} / \mathrm{cm}^{2}$. Even though our model clearly explains the observed SH field profiles as well as the experimentally measured magnitudes of the nonlinear phase shifts, the sign of the latter could only be reproduced by arbitrarily changing the core refractive index at the second harmonic frequency. The latter observation clearly deserves further scrutiny. We believe that well controlled fibers with improved materials will lead to even larger nonlinear phase shifts which could be ap- plied to phenomena requiring Kerr-law nonlinear responses at low input powers.

\section{Acknowledgements}

This research was supported by AFOSR (91-0339) and ARPA and ARO(DAAL03-91-C-00420) at C.R.E.O.L.. W.T. acknowledges the support, while at C.N.E.T., of a N.S.F./N.A.T.O. postdoctoral fellowship. The research of G.K. has been made possible by 
a fellowship of the Royal Netherlands Academy of Arts and Sciences. We also acknowledge $H$. Hoekstra of the University of Twente for implementing important parts of the SHGBPM code.

\section{References}

[1] Chr. Flytzanis, N. Bloembergen, Prog. Quantum Electron. 4 (1976) 271.

[2] G. Meredith, Phys. Rev. B 24 (1981) 5522.

[3] E. Yablonovitch, C. Flytzanis, N. Bloembergen, Phys. Rev. Lett. 29 (1972) 865.

[4] R. DeSalvo, D.I. Hagan, M. Sheik-Bahae, G.I. Stegeman, E.W. Van Stryland, H. Vanherzeele, Optics Lett. 17 (1992) 28.

[5] T.F. Carruthers, I.N. Duling III, Optics Lett. 15 (1990) 804.

[6] G.I. Stegeman, M. Sheik-Bahae, E. Van Stryland, G. Assanto, Optics Lett. 18 (1993) 13.

[7] G. Assanto, G.I. Stegeman, M.S. Sheik-Bahae, E.W. Van Stryland, Appl. Phys. Lett. 62 (1993) 1323.

[8] M.L. Sundheimer, Ch. Bosshard, E. Van Stryland, G.I. Stegeman, Optics Lett. 18 (1993) 1397.

[9] A.E. Kaplan, Optics Lett. 18 (1993) 1223.

[10] D. Danielius, P. Di Trapani, A. Dubietis, A. Piskaskas, D. Podenas, G.P. Banfi, Optics Lett. 18 (1993) 574.

[11] D.C. Hutchings, J.S. Aitchison, C.N. Ironside, Optics Lett. 18 (1993) 793.

[12] C.J. McKinstrie, D.F. DuBois, Phys. Rev. Lett. 57 (1986) 2022.

[13] G. Kalocsai, J.W. Haus, Optics Comm. 97 (1993) 239.

[14] R. Schiek, J. Opt. Soc. Am. B 10 (1993) 1848.

[15] M.J. Werner, P.D. Drummond, Optics Lett. 10 (1993) 2390.

[16] G.R. Meredith, Chem. Phys. Lett. 92 (1982) 165.

[17] S. Mukamel, Z. Deng, J. Grad, J. Opt. Soc. Am. B 5 (1988) 804.
[18] W.E. Torruellas, R. Zanoni, G.I. Stegeman, G.R. Mohlmann, E.W.P. Erdhuisen, W.H.G. Horsthuis, J. Chem. Phys. 94 (1991) 6851.

[19] J.H. Andrews, K.L. Kowalski, K.D. Singer, Phys. Rev. A 46 (1992) 4172.

[20] D.S. Chemla and J. Zyss, Nonlinear Optical Properties of Organic Molecules and Crystals (Academic, London, 1987).

[21 ] I. Ledoux, C. Lepers, A. Perigaud, J. Badan, J. Zyss, Optics Comm. 80 (1990) 149.

[22] S. Ducharme, W.P. Risk, W.E. Moerner, V.Y. Lee, R.J. Twieg, G.C. Bjorklund, Appl. Phys. Lett. 57 (1990) 537.

[23] P.V. Vidakovic, M. Coquillay, F. Salin, J. Opt. Soc. Am. B 4 (1987) 998.

[24] M. Yamashita, K. Torizuka, T. Uemiya, Appl. Phys. Lett. 57 (1990) 1301.

[25] D.Y. Kim, W.E. Torruellas, J. Kang, C. Bosshard, G.I. Stegeman, P. Vidakovic, J. Zyss, W.E. Moerner, R. Twieg, G. Bjorklund, Optics Lett. 19 (1994) 868.

[26] P. Kerkoc, M. Zgonik, K. Sutter, Ch. Bosshard, P. Gunter, J. Opt. Soc. Am. B 7 (1990) 313;

Optical Glass Catalog, (Schott Glass Technologies Inc., Duryea, Pennsylvania, 1992).

[27] See for example, K. Chikuma, S. Umegaki, J. Opt. Soc. Am. B 9 (1992) 1083.

[28] T. Kondo, R. Ito, in: Molecular Nonlinear Optics: Materials, Physics, and Devices, ed. J. Zyss (Academic, New York, 1994).

[29] D. Marcuse, Theory of Dielectric Optical Waveguides (Academic, New York, 1991).

[30] J. Sipe, G.I. Stegeman, J. Opt. Soc. Am. 69 (1979) 1676.

[31] V.C.Y. So, R. Normandin, G.I. Stegeman, J. Opt. Soc. Am. 69 (1979) 1166.

[32] H.J.W.M. Hoekstra, G.J.M. Krijnen, P.V. Lambeck, Optics Comm. 97 (1993) 301.

[33] G.J.M. Krijnen, H.J.W.M. Hoekstra, P.V. Lambeck, Proceedings of ECIO-93, 5-4, Neuchatel, 1993.

[34] T. Uemiya, N. Uenishi, S. Okamoto, K. Chikuma, K. Kumata, T. Kondo, R. Ito, S. Umegaki, Appl. Optics 31 (1992) 7581. 